\title{
Detailed theoretical and experimental investigation of high-gain erbium-doped fiber amplifier
}

Pedersen, Bo; Dybdal, Kristen; Dam-Hansen, Carsten; Bjarklev, Anders Overgaard; Povlsen, Jørn Hedegaard; Vendeltorp-Pommer, Helle; Larsen, Carl Christian

Published in:

I E E E Photonics Technology Letters

Link to article, DOI:

10.1109/68.62011

Publication date:

1990

Document Version

Publisher's PDF, also known as Version of record

Link back to DTU Orbit

Citation $(A P A)$ :

Pedersen, B., Dybdal, K., Dam-Hansen, C., Bjarklev, A. O., Povlsen, J. H., Vendeltorp-Pommer, H., \& Larsen, C. C. (1990). Detailed theoretical and experimental investigation of high-gain erbium-doped fiber amplifier. I $E E E$ Photonics Technology Letters, 2(12), 863-865. https://doi.org/10.1109/68.62011

\section{General rights}

Copyright and moral rights for the publications made accessible in the public portal are retained by the authors and/or other copyright owners and it is a condition of accessing publications that users recognise and abide by the legal requirements associated with these rights.

- Users may download and print one copy of any publication from the public portal for the purpose of private study or research.

- You may not further distribute the material or use it for any profit-making activity or commercial gain

- You may freely distribute the URL identifying the publication in the public portal 


\title{
Detailed Theoretical and Experimental Investigation of High-Gain Erbium-Doped Fiber Amplifier
}

\author{
BO PEDERSEN, KRISTEN DYBDAL, CARSTEN DAM HANSEN, ANDERS BJARKLEV, \\ JØRN HEDEGAARD POVLSEN, HELLE VENDELTORP-POMMER, AND \\ CARL CHRISTIAN LARSEN
}

\begin{abstract}
A full scale numerical model for the erbium-doped fiber amplifier has been developed, incorporating realistic index and erbiumconcentration profiles as well as the spectral distribution of amplified spontaneous emission. The high accuracy of the model is demonstrated by comparison with a comprehensive set of data, including gain, ASE, and pump power, obtained for a well characterized Er-Al-doped fiber. An absorption to emission cross section ratio of 1.0 was measured at the gain peak. Pumping at $654 \mathrm{~nm}$, the excited state absorption was observed to be insignificant. $A$ high gain of $39.6 \mathrm{~dB}$ was achieved in the experiment.
\end{abstract}

\section{INTRODUCTION}

$\mathrm{T}$ HEORETICAL work on erbium-doped fiber amplifiers has so far been based on simplifying assumptions such as small signal power, small amplified spontaneous emission (ASE) power, step profiles of the index, and erbium-concentration [1]-[3]. Most of these assumptions do not strictly apply to real fibers with high gain and output power. Therefore, adjustable parameters are required to obtain agreement with experiment. Thus, for applications where quantitative predictions are important, full scale calculations based on realistic index and erbium-concentration profiles as well as ASE spectral power distribution are necessary.

The present letter describes a detailed numerical model taking full account of the real $\mathrm{LP}_{01}$-mode profiles and the spectral power distribution of the ASE. In order to assess the accuracy of the model, calculations are compared to results of a comprehensive experimental investigation of a particular Er-Al-doped fiber. All basic parameters characterizing the fiber, i.e., refractive index profile, erbium-concentration profile, intrinsic loss, emission and absorption cross sections, and fluorescence lifetime are experimentally determined to provide realistic input parameters to the model. The agreement between calculations and measurements is observed to be excellent over more than four orders of magnitude.

Manuscript received June 20, 1990; revised September 27, 1990. This work was supported by the Danish Technical Research Council and the National Agency of Industry and Trade, Denmark.

B. Pedersen, C. D. Hansen, A. Bjarklev, J. H. Povlsen, and H. Vendeltorp-Pommer are with the Electromagnetics Institute, CBT, Technical University of Denmark, 2800 Lyngby, Denmark.

K. Dybdal is with Jydsk Telefon, 8310 Tranbjerg, Denmark.

C. C. Larsen is with Lycom A/S, 2605 Brøndby, Denmark.

IEEE Log Number 9041160.

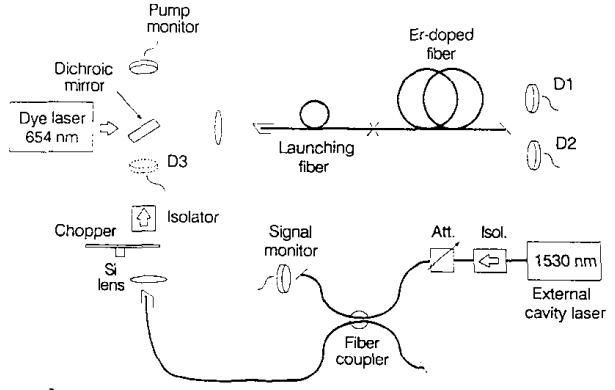

Fig. 1. $\mathrm{Er}^{3+}$-fiber amplifier: experimental setup for measurement of gain, attenuated pump power, forward and backward ASE power.

\section{EXPERIMENT}

The properties of the fiber amplifier were measured in a setup as shown in Fig. 1. The coupled pump $\left(\lambda_{p}=654 \mathrm{~nm}\right)$ and signal $\left(\lambda_{s}=1530 \mathrm{~nm}\right)$ powers in the undoped launching fiber were accurately calibrated to the monitor detectors. A low back-reflection of $-57 \mathrm{~dB}$ from the launching setup into the erbium-doped fiber was measured. The erbium-doped fiber was fusion spliced to the launching fiber and terminated with a cut at an oblique angle. Splice losses at signal and pump wavelengths were measured after each series of measurements. Losses were low, typically 0.3-0.7 dB.

The residual pump power and forward and backward travelling ASE powers were measured in calibrated detectors $D 1, D 2$, and $D 3$, respectively. Removing $D 3$ from the signal beam path the output signal was measured with $D 2$ connected to a lock-in amplifier (input signal $<-46 \mathrm{dBm}$ ). The results are estimated to be accurate to within $\pm 0.3 \mathrm{~dB}$.

The erbium-doped fiber was prepared by the solution-doping technique [4] with aluminum as the index-raising element. The fiber had an outer diameter of $75 \mu \mathrm{m}$. The dopant concentration profiles of $\mathrm{Al}$ and Er were measured in a slice of the preform by scanning electron microprobe spectroscopy. The attenuation spectrum and the fluorescence spectrum and lifetime were measured by standard techniques [5].

THEORY

The present model constitutes an extension of the model described in detail in [6] and is based on a three-level laser 
system with a fourth level added due to the excited state absorption. The population in the ground state, $n_{1}$, and in the excited state $n_{2}$ at any point $(r, \phi, z)$ in the fiber are determined by the combined influence of the local intensities of pump, signal and foward ( + ) and backward ( - ) ASE [6]:

$$
\begin{aligned}
& n_{2}(r, \phi, z) \\
& \quad=\frac{W_{13}(r, \phi, z)+W_{12}(r, z)}{W_{13}(r, \phi, z)+W_{12}(r, z)+W_{21}(r, z)+A_{21}}
\end{aligned}
$$

where the pumping rate $W_{13}$ is calculated by summation over the pump modes and $A_{21}$ is the spontaneous emission rate [6]. Rather than using the equivalent bandwidth approximation of [6], the stimulated emission $W_{21}$ and absorption $W_{12}$ rates are calculated by integration over the ASE spectral power density $S_{\mathrm{ASE}}$ :

$$
\begin{aligned}
& W_{i j}(r, z)=\left[\frac{\sigma_{i j}\left(v_{s}\right)}{h v_{s}} P_{s}(z)\right. \\
& \left.+\int_{0}^{\infty} \frac{\sigma_{i j}(v)}{h v}\left(S_{\mathrm{ASE}}^{+}(v, z)+S_{\mathrm{ASE}}^{-}(v, z)\right) d v\right] \cdot I_{s}^{01}(r)
\end{aligned}
$$

where $v$ denotes optical frequency, $I^{01} s(r)$ is the normalized signal $\mathrm{LP}_{01}$-mode intensity [7] and $\sigma_{i j}$ is the wavelength dependent emission ( $i j=21)$ or absorption $(i j=12)$ cross section. $P_{s}$ is the signal power.

The propagation of pump, signal, and ASE are calculated from well known differential equations [6], e.g.,

$$
\begin{aligned}
& \frac{d S_{\mathrm{ASE}}^{ \pm}(v, z)}{d z}= \pm 2 h v \gamma_{21}(v) \\
& \pm\left(\gamma_{21}(v)-\gamma_{12}(v)\right) S_{\mathrm{ASE}}^{ \pm}(v, z)
\end{aligned}
$$

where the emission- or absorption-factor $\gamma_{i j}$ is determined from the cross section $\sigma_{i j}$ and from the overlap integral between the signal $\mathrm{LP}_{01}$-mode and the population concentration in the excited or ground state [6]. The calculation of the pump propagation further includes the erbium-independent attenuation (see below). The ASE powers are calculated by spectral integration of $S_{\mathrm{ASE}}(v)$.

The solution is obtained by simultaneous numerical integration of (3) for $S_{\mathrm{ASE}}^{+}(v, z)$ and $S_{\mathrm{ASE}}^{-}(v, z)$, evaluated in 100 frequencies with a spacing of $200 \mathrm{GHz}$, and the analogous equations for $P_{s}(z)$ and $P_{p}(z)$. At each point $z$ along the fiber population inversion over the fiber cross section is obtained from (1) and (2) using pump and signal mode profiles calculated [7] from the measured index profile. The overlap integrals of $\gamma_{i j}$ are subsequently computed on the basis of the measured erbium profile by numerical integration of the radial dependence. The azimuthal integration is performed analytically.

To fulfil the boundary conditions at both fiber ends, $z=$ $0, L$, an iterative procedure, based on forward and backward integration through the fiber, was adopted. The starting point of each forward integration is the input powers $P_{s}(0), P_{p}(0)$, $S_{\mathrm{ASE}}^{+}(v, 0)$ and the result of the previous backward integration as the best guess for $S_{\mathrm{ASE}}^{-}(v, 0)$. At the first pass $S_{\mathrm{ASE}}^{-}(v, 0)$ is clamped at zero throughout the fiber. Analogously, the re- sults for $P_{s}(L), P_{p}(L)$ and $S_{\mathrm{ASE}}^{+}(v, L)$, together with the boundary condition $S_{\mathrm{ASE}}^{-}(v, L)=0$ are taken as starting point for the following backward integration. Typically, ten iterations are necessary depending on the actual conditions.

\section{Results}

The shape of the measured index and erbium profiles were found to be identical and very well approximated by an $\alpha$-profile $\left(C\left(1-(r / a)^{\alpha}\right)\right.$ with $\alpha=3$ and a core radius of $a=2.7 \mu \mathrm{m}$. Consequently, this parametrization was used as a convenient representation of the data in the calculations. The absolute scale was measured to be $C=10^{-2}$ for the index difference and $C=2.0 \times 10^{24} \mathrm{~m}^{-3}$ for the erbium concentration, the latter value with an estimated uncertainty of $10 \%$ due to calibration uncertainties. The calculated $\mathrm{LP}_{11}$ cutoff wavelength is $947 \mathrm{~nm}$ in agreement with the measured value of $960 \mathrm{~nm}$.

Emission and absorption cross sections are calculated, as shown in [5] from the measured fluorescence and attenuation spectra, using a measured fluorescence lifetime of $10.6 \mathrm{~ms}$ and including an empirical correction factor of 1.15 for the absorption [5]. As an example, the emission cross section as well as the absorption cross section were found to be $5.0 \times$ $10^{-25} \mathrm{~m}^{2}$ at the signal wavelength, $\lambda_{s}=1530 \mathrm{~nm}$. The resulting cross section ratio of $\sigma_{12} / \sigma_{21}=1.0$ is supported by the close agreement between the measured asymptotic gain for heavily pumped fibers and the measured (unpumped) fiber attenuation of $2.14 \mathrm{~dB} / \mathrm{m}$ at $1530 \mathrm{~nm}$, in contrast to the results of [8]. Based on the fiber attenuation and the absorption cross section a maximum value for the erbium concentration of $C=2.1 \times 10^{24} \mathrm{~m}^{-3}$ is calculated in very good agreement with the measured value. The calculated result is adopted since it is considered more accurate.

Knowing the erbium concentration and the attenuation at the pump wavelength $\lambda_{p}=654 \mathrm{~nm}$, the absorption cross section is found to be $\sigma_{13}=3.2 \times 10^{-25} \mathrm{~m}^{2}$. Furthermore, from the attenuation spectrum the fiber was observed to have an intrinsic background loss of $0.3 \mathrm{~dB} / \mathrm{m}$. The excited state absorption cross section $\sigma_{24}$ was extracted from the pump attenuation in a heavily pumped $5 \mathrm{~m}$ long fiber corrected for the intrinsic attenuation. An upper limit of $0.06 \times 10^{-25} \mathrm{~m}^{2}$ was extracted, somewhat lower than previously reported [9]. In the calculations $\sigma_{24}=0$.

Visual inspection of the output pump far field indicated that only the $\mathrm{LP}_{01}$ pump mode was excited. The intensity in the only other allowed mode $\mathrm{LP}_{11}$ was consequently set to zero. Still, trial calculations show that even $25 \%$ of the pump power in this mode will influence the results by less than 0.5 $\mathrm{dB}$.

The model predictions are compared with the experimental results in Fig. 2 for the small signal gain and in Fig. 3 for the residual pump power. As observed, the agreement is excellent over more than four orders of magnitude of the gain. The measured maximum gain of $39.6 \mathrm{~dB}$ is reached with a fiberlength of $30 \mathrm{~m}$ and a launched pump power of $115 \mathrm{~mW}$. The residual pump power tends to be underestimated by the calculations on fiberlengths beyond $20 \mathrm{~m}$. This is attributed to minor inaccuracies in the input parameters. 


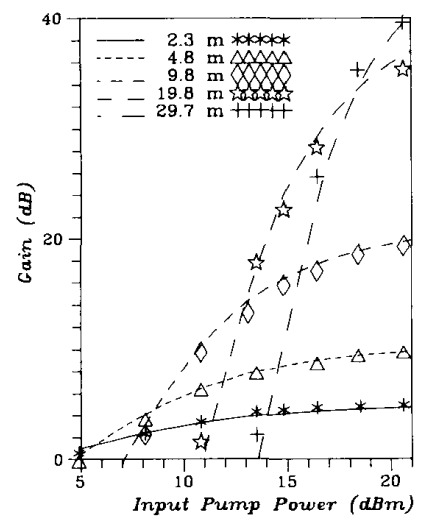

Fig. 2. Comparison of measured (symbols) and calculated gains for different lengths of fibre as a function of launched pump power.

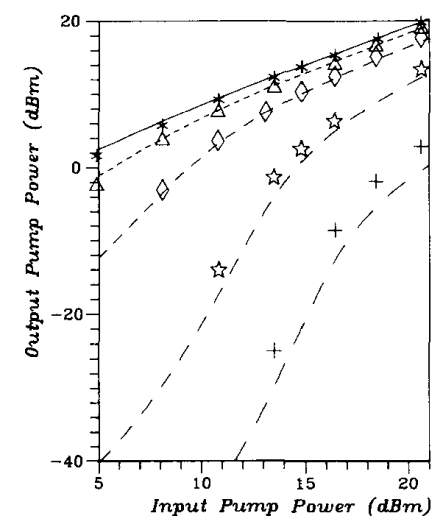

Fig. 3. Comparison of measured (symbols) and calculated output pump power as a function of fibre length and launched pump power.

Furthermore, an excellent agreement between measured and calculated forward (a) and backward (b) ASE powers is observed in Fig. 4. An equally good agreement was observed between measured and calculated forward and backward ASE spectra. This will allow straightforward calculation of noise characteristics at arbitrary signal wavelengths.

The present model constitute a significant improvement of accuracy and generality as compared to previous calculations [2], [3], which have to incorporate adjustable parameters in order to match experimental results. This improvement is attributed to the proper treatment of the guided-light and erbium concentration profiles. Also, using the correct spectral shapes of the emission and absorption cross sections, it is unnecessary to introduce an effective optical bandwidth, the value of which depends on the actual experimental conditions.

\section{CONCLUSION}

A model has been developed, which is able to provide a comprehensive and highly accurate description of the characteristics of erbium-doped fiber amplifiers, without having to incorporate adjustable parameters. A model with this accuracy will be valuable in the design and optimization of practical fiber amplifier.

The erbium-doped fiber was found to have an absorption to emission cross section ratio of 1.0 at the gain peak and low
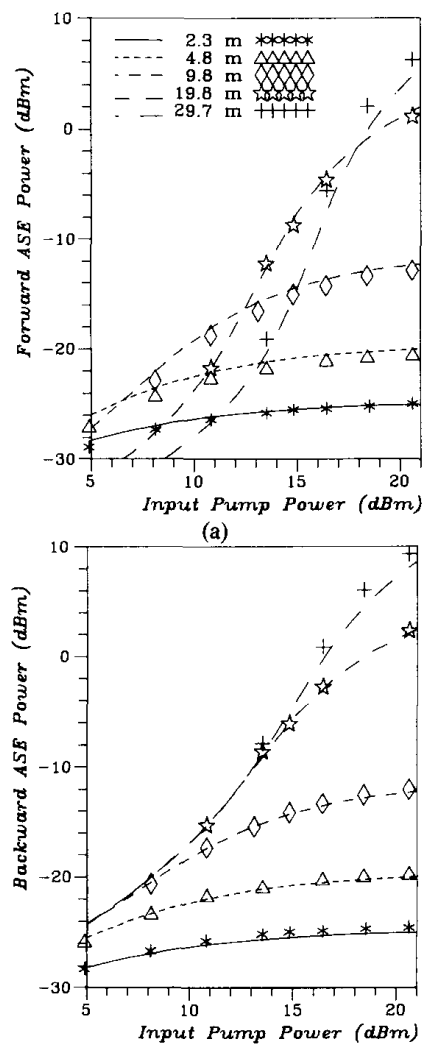

Fig. 4. Comparison of measured (symbols) and calculated ASE power for different fiber lengths and launched pump power.

excited state absorption at the pump wavelength of $654 \mathrm{~nm}$. A high gain of $39.6 \mathrm{~dB}$ was achieved.

\section{ACKNOWLEDGMENT}

The authors would like to thank Dr. E. Nicolaisen for fruitful discussions and suggestions.

\section{REFERENCES}

[1] J. R. Armitage, "Three-level fiber laser amplifier: A theoretical model," Appl. Opt., vol. 27, p. 4831, 1988.

[2] E. Desurvire, C. R. Giles, and J. R. Simpson, "Gain saturation effects in high-speed, multichannel erbium-doped fiber amplifiers at $\lambda=1.53 \mu \mathrm{m}$," $J$. Lightwave Technol., vol. LT-7, p. 2095, 1989.

[3] P. R. Morkel and R. I. Laming, "Theoretical modeling of erbiumdoped fiber amplifiers with excited-state absorption," Opt. Lett., vol. 14, p. $1062,1989$.

[4] S. B. Poole, "Fabrication of Al203 co-doped optical fibers by a solution-doping technique," in Proc. ECOC, 1988, p. 433.

[5] K. Dybdal, N. Bjerre, J. Engholm Pedersen, and C. C. Larsen, "Spectroscopic properties of Er-doped silica fibers and preforms," SPIE, vol. 1171, Fibers, Fiber Laser Sources and Amplifiers, p. 209, 1989.

[6] A. Bjarklev, S. Lomholt Hansen, and J. Hedegaard Povlsen, “Large signal modeling of an Erbium doped fiber amplifier," SPIE, vol. 1171, Fibers, Fiber Laser Sources and Amplifiers, p. 118, 1989.

[7] P. L. Danielsen, "Analytical expressions for group delay in the far field from an optical fibre having an arbitrary index profile," IEEE $J$. Quantum Electron., vol. QE-19, p. 850, 1981.

[8] W. L. Barnes, R. I. Laming, P. R. Morkel, and E. J. Tarbox, "Absorption-emission cross section ratio for $\mathrm{Er}^{3+}$ doped fibers at 1.5 $\mu \mathrm{m}, "$ in CLEO'90, OSA Tech. Dig. Series, vol. 7, p. 50, 1990.

[9] R. I. Laming, S. B. Poole, and J. E. Tarbox, "Pump excited-state absorption in erbium-doped fibers," Opt. Lett., vol. 13, p. 1084 1988. 